\title{
Dihydroartemisinin-induced apoptosis in human acute monocytic leukemia cells
}

\author{
JIA-TIAN CAO $^{1^{*}}$, HUI-MIN MO $^{2,3^{*}}$, YUE WANG $^{1 *}$, KAI ZHAO $^{2,3}$, TIAN-TIAN ZHANG ${ }^{1}$, \\ CHANG-QIAN WANG ${ }^{1}$, KAI-LIN XU ${ }^{2,3}$ and ZHI-HUA HAN ${ }^{1}$ \\ ${ }^{1}$ Department of Cardiology, The Ninth People's Hospital, Shanghai Jiaotong University Medical School, \\ Shanghai 200011; ${ }^{2}$ Institute of Hematology, Xuzhou Medical University; ${ }^{3}$ Department of Hematology, \\ The Affiliated Hospital of Xuzhou Medical University, Xuzhou, Jiangsu 221002, P.R. China
}

Received May 23, 2016; Accepted August 8, 2017

DOI: 10.3892/ol.2017.7644

\begin{abstract}
Dihydroartemisinin (DHA) is a derivative of artemisinin. The present study aimed to investigate whether DHA induces apoptosis in the THP-1 human acute monocytic leukemia cell line (AMoL), and to identify the relative molecular mechanisms. The results of the present study demonstrated that the viability of THP-1 cells were inhibited by DHA in a dose- and time-dependent manner, which was accompanied by morphological characteristics associated with apoptosis. After $24 \mathrm{~h}$ of $200 \mu \mathrm{M}$ DHA treatment, the proportion of apoptotic cells was significantly increased compared with the untreated controls $(\mathrm{P}<0.01)$. In addition, DHA downregulated the levels of B-cell lymphoma (Bcl)-2, protein kinase B (Akt)1, $\mathrm{Akt} 2$ and $\mathrm{Akt} 3$ gene expression, and increased the expression of the $\mathrm{Bcl}-2$-associated $\mathrm{X}$ protein apoptosis regulator. The protein expression of phospho-Akt and phospho-extracellular signal-regulated kinase (ERK) was also decreased, and the protein expression level of cleaved caspase-3 was increased following treatment with DHA. Therefore, DHA may induce apoptosis in the AMoL THP-1 cell line via currently unknown underlying molecular mechanisms, including the downregulation of ERK and Akt, and the activation of caspase-3.
\end{abstract}

Correspondence to: Professor Zhi-Hua Han, Department of Cardiology, The Ninth People's Hospital, Shanghai Jiaotong University Medical School, 639 Zhizaoju Road, Shanghai 200011, P.R. China

E-mail: doctorhanzhihua@163.com

Professor Kai-Lin Xu, Institute of Hematology, Xuzhou Medical University, 99 West Huaihai Road, Xuzhou, Jiangsu 221002, P.R. China

E-mail: lihmd@163.com

*Contributed equally

Key words: dihydroartemisinin, acute monocytic leukemia, apoptosis, extracellular signal-regulated kinase, protein kinase B, caspase-3

\section{Introduction}

Acute myeloid leukemia (AML) is a clonal disorder that comprises a group of clonal malignant diseases, in which the cancer cells originate from the bone marrow and display genetic instability, leading to the accumulation of abnormal immature myeloid cells in the bone marrow and blood (1). The principal therapeutic strategies for patients with AML are aggressive chemotherapeutic regimens and hematopoietic stem cell transplantation (HSCT) (1-3). However, AML consists of 8 subtypes, including $\mathrm{M}_{0}$ (minimally differentiated AML), $\mathrm{M}_{1}$ (AML without maturation), $\mathrm{M}_{2}$ (AML with maturation), $\mathrm{M}_{3}$ (acute promyelocytic leukemia), $\mathrm{M}_{4}$ (acute myelomonocytic leukemia), $\mathbf{M}_{5}$ [acute monocytic leukemia (AMoL)], $\mathbf{M}_{6}$ (erythroleukemia) and $\mathbf{M}_{7}$ (acute megakaryoblastic leukemia). Patients with AML who receive intensive chemotherapy may achieve complete remission; however, the overall survival rate of patients with AML is poor and the treatment is typically associated with serious complications (4). In addition, there is no optimal therapeutic schedule for each type of AMoL (5). Therefore, the aim of the present study was to identify a novel drug for treating $\mathrm{M}_{5}(\mathrm{AMoL})$.

The development of AMoL is a complex, multistep and multifactorial process. Features of $\mathrm{M}_{5}$ typically include increased numbers of white blood cells, an increased rate of marrow infiltration and an increase in chromosomal aberrations including translocation, mutation, aneuploidy and the formation of fusion genes $(6,7)$. Furthermore, the clinical complete remission rate is low with poor prognosis. A total of 87 different translocations have been identified, with 11 q23 chromosomal abnormalities accounting for $\sim 22 \%$ of $\mathrm{M}_{5}$ cases. There are various chemotherapy regimens used to treat patients with AMoL including: i) Homoharringtonine, cytarabine and etoposide; ii) daunorubicin, Ara-c and teniposide; and iii) mitoxantrone, Ara-c and teniposide (7). However, $\mathrm{AMoL}$ is insensitive to a number of chemotherapy regimens, thus patients with AMoL exhibit a low remission rate and decreased survival time $(2,6,7)$. Therefore, novel effective drugs are required.

Since novel antitumor drugs fell under investigation, bioactive natural products have emerged and gained considerable attention. Artemisinin is an effective drug for treating 
malaria and belongs to the family of sesquiterpene lactones, which are produced by the Artemisia annua plant (8). Dihydroartemisinin (DHA) is a water-soluble semi-synthetic derivative of artemisinin (9), and it is commercially combined with piperaquine as an effective therapy for malaria with limited side effects $(10,11)$. In addition, DHA has been identified to exhibit inhibitory effects on cancer cells, including lung carcinoma (12), osteosarcoma (13) and ovarian cancer (14). Previous studies have demonstrated that DHA may inhibit molt-4 acute lymphoid cell leukemia cells $(15,16)$. However, the effects and underlying mechanisms of action of DHA in AMoL remain unknown.

Therefore, the aim of the present study was to analyze the antitumor effects of DHA against AMoL and to investigate its underlying biological mechanisms. An in vitro approach (Fig. 1A) was used to elucidate the molecular mechanisms underlying these effects of DHA. The results of the present study verified that DHA exhibited effective antitumor activity against $\mathrm{AMoL}$ in vitro via the downregulation of phospho-protein kinase B (p-Akt) and p-extracellular signal-regulated kinase (ERK) protein expression, and the upregulation of cleaved caspase-3 levels.

\section{Materials and methods}

Reagents and antibodies. The chemical reagents, including DHA, used in the present study were purchased from Sigma-Aldrich (Merck KGaA, Darmstadt, Germany) unless stated otherwise. A stock solution $(200 \mathrm{mM})$ of DHA was prepared in dimethyl sulfoxide (Sigma-Aldrich; Merck $\mathrm{KGaA}$ ) and stored at $-20^{\circ} \mathrm{C}$. Primary antibodies against p-ERK (cat. no. 4348S), total (t)-ERK (cat. no. 4695S), p-Akt (cat. no. 4060S), t-Akt (cat. no. 4685S), cleaved caspase-3 (cat. no. 1050S), $\beta$-actin (cat. no. 4970S) and secondary antibodies (cat. no. 7074) were purchased from Cell Signaling Technology, Inc. (Danvers, MA, USA).

Cell culture. The THP-1 human acute monocytic leukemia cell line was purchased from the Chinese Academy of Sciences (Shanghai, China). THP-1 cells were cultured in RPMI-1640 (HyClone; GE Healthcare, Logan, UT, USA) supplemented with $10 \%$ fetal bovine serum, $100 \mathrm{U} / \mathrm{ml}$ penicillin and $100 \mu \mathrm{g} / \mathrm{ml}$ streptomycin (all from HyClone; GE Healthcare) at $37^{\circ} \mathrm{C}$ in a humidified atmosphere containing $5 \% \mathrm{CO}_{2}$. All cells were used within 20 passages.

Cell viability assay. DHA cytotoxicity was determined using a Cell Counting Kit-8 (CCK-8; Dojindo Molecular Technologies, Inc., Kumamoto, Japan), which evaluated the metabolic activity of viable cells. A total of 20,000 cells/well were seeded into 96-well plates in RPMI-1640 medium, which contained $100 \mathrm{nM}$ phorbol 12-myristate 13-acetate to induce adherence as THP-1 cells were suspending cells, and subsequently treated with $0,25,50,100,150$ or $200 \mu \mathrm{M}$ DHA for $24,48,72$ and $96 \mathrm{~h}$ at $37^{\circ} \mathrm{C}$. Following treatment with DHA for 24, 48, 72 and $96 \mathrm{~h}$, the RPMI-1640 medium was removed and the cells were washed with PBS. Subsequently, $100 \mu \mathrm{l}$ RPMI-1640 medium and $10 \mu \mathrm{l}$ CCK-8 solution was added to each well and incubated at $37^{\circ} \mathrm{C}$ for $2.5 \mathrm{~h}$. The optical density (OD) at $450 \mathrm{~nm}$ was determined daily for the following
4 days using a microplate reader (BioTek Instruments, Inc., Winooski, VT, USA). All measurements were repeated in triplicate. The cell viability compared with the control group was calculated using the following equation: Cell viability to control $(\%)=\mathrm{OD}_{\text {drug-treated group }} / \mathrm{OD}_{\text {control group }}$.

Apoptosis analysis. Cells were seeded at $1 \times 10^{6}$ cells/well in 6-well plates and harvested $24 \mathrm{~h}$ after treatment with 0 , $25,50,100,150$ and $200 \mu \mathrm{M}$ DHA. Cells were centrifuged at $500 \mathrm{x} \mathrm{g}$ for $5 \mathrm{~min}$ at $4^{\circ} \mathrm{C}$ and the supernatants were discarded. Subsequently, the cells were resuspended in $1 \mathrm{X}$ annexin-binding buffer (BD Biosciences, Franklin Lakes, NJ, USA). Apoptotic cells were determined using annexin V-fluorescein isothiocyanate and propidium iodide staining (BD Biosciences, Franklin Lakes, NJ, USA) and quantified using a flow cytometer (BD Biosciences). FlowJo software (version 7.6.1; FlowJo LLC, Ashland, OR, USA) was used to analyze the rate of apoptosis. The cell proliferation results presented in Fig. $1 \mathrm{~B}$ and $\mathrm{C}$ demonstrate the inhibitory effect of DHA on THP-1 cell proliferation. Therefore, further analysis was used to determine whether DHA is able to induce apoptosis. In Fig. 2A, the lower right quadrant represents early apoptosis, whereas the upper right quadrant represents late apoptosis. The proportion of apoptotic cells identified includes cells in early and late apoptosis.

Reverse transcription-quantitative polymerase chain reaction (RT-qPCR). THP-1 cells $\left(1 \times 10^{6}\right.$ cells/well) were seeded at in 6 -well plates and harvested following treatment with $0,25,50$, 100,150 and $200 \mu \mathrm{M}$ DHA for $24 \mathrm{~h}$. Cells were centrifuged at $500 \mathrm{x} \mathrm{g}$ for $5 \mathrm{~min}$ at $4^{\circ} \mathrm{C}$ and the supernatants were discarded. Total RNA was isolated from THP-1 cells treated with 0 , $25,50,100,150$ and $200 \mu \mathrm{M}$ DHA using the AxyPrep ${ }^{\mathrm{TM}}$ Multisource Total RNA Miniprep kit (Axygen Scientific, Inc., Union City, CA, USA). Equivalent amounts of RNA were converted into cDNA using the PrimeScript ${ }^{\mathrm{TM}}$ RT Reagent kit (Takara Bio, Inc., Otsu, Japan) at $37^{\circ} \mathrm{C}$ for $15 \mathrm{~min}$ and $85^{\circ} \mathrm{C}$ for $5 \mathrm{sec}$. cDNA was measured using a Nanodrop 2000 instrument (Thermo Fisher Scientific, Waltham, MA, USA). qPCR was performed using an ABI 7500 Sequencing Detection System and SYBR ${ }^{\circledR}$ Premix Ex Taq (Takara Bio, Inc.). All procedures were performed according to the manufacturers' protocols. Cycling conditions included 40 cycles of $95^{\circ} \mathrm{C}$ for $5 \mathrm{sec}$ and $60^{\circ} \mathrm{C}$ for $34 \mathrm{sec}$. Gene expression was quantified using the $2^{-\Delta \Delta \mathrm{Cq}}$ method (17). $\beta$-actin was used as the reference gene and all primer sequences are listed in Table I.

Western blot analysis. THP-1 cells were analyzed using qPCR and cells were lysed in lysis buffer containing complete EDTA-free tablets which are a protease inhibitor cocktail containing phenylmethylsulfonyl fluoride, aprotinin, bestatin, E-64, leupeptin and pepstatin A (Roche Applied Science, Penzbery, Germany). The protein concentration was quantified using the bicinchoninic acid protein assay kit (Santa Cruz Biotechnology, Inc., Dallas, TX, USA). For western blot analysis, $20 \mu \mathrm{g}$ total protein were boiled and subsequently separated by SDS-PAGE using a $10 \%$ gel for p-Akt, total (t-) Akt, p-ERK, t-ERK and $\beta$-actin and a $12.5 \%$ gel for cleaved caspase-3. Following electrophoresis, proteins were blotted onto polyvinylidene difluoride membranes and 
A

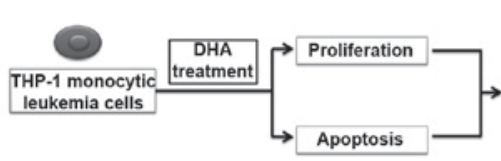

C

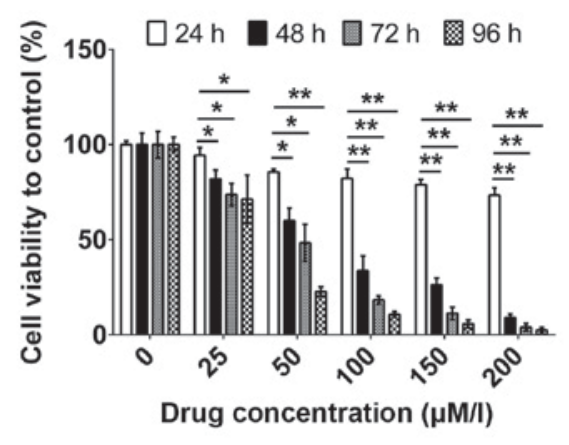

B

x40 Molecular
mechanisms

$\times 100$

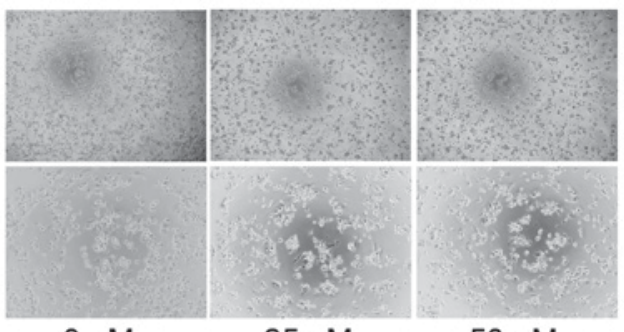

$0 \mu \mathrm{M}$

$25 \mu \mathrm{M}$

$50 \mu \mathrm{M}$

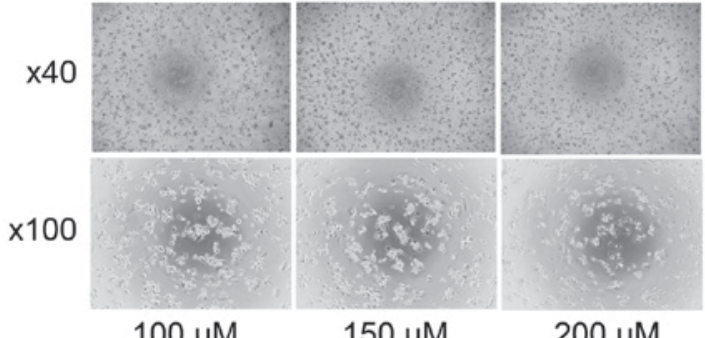

Figure 1. Proliferation inhibition of THP-1 cells following treatment with DHA. (A) Schematic of the approach used in this study. (B) The morphology of THP-1 cells at $\mathrm{x} 40$ and $\mathrm{x} 100$ following $0,25,50,100,150$ and $200 \mu \mathrm{M}$ DHA treatment. (C) CCK-8 assay indicated that DHA could inhibit the proliferation of THP-1 cells in a dose- and time-dependent manner. Data are presented as the mean \pm standard deviation. ${ }^{* *} \mathrm{P}<0.01,{ }^{*} \mathrm{P}<0.05$. All data were obtained from at least three independent experiments. DHA, dihydroartemisinin; CCK-8, Cell Counting Kit-8.

A

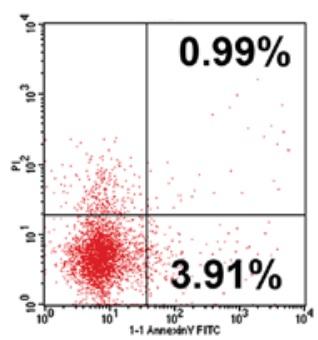

$0 \mu \mathrm{M}$

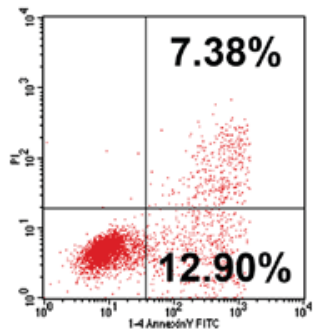

$100 \mu \mathrm{M}$

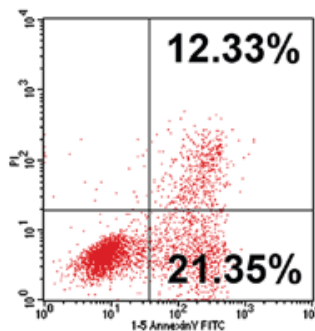

$150 \mu \mathrm{M}$

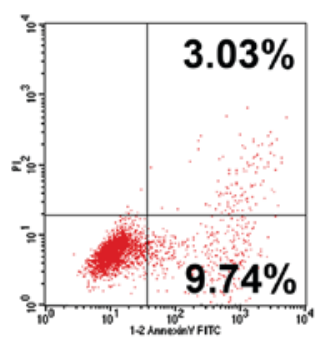

$25 \mu \mathrm{M}$
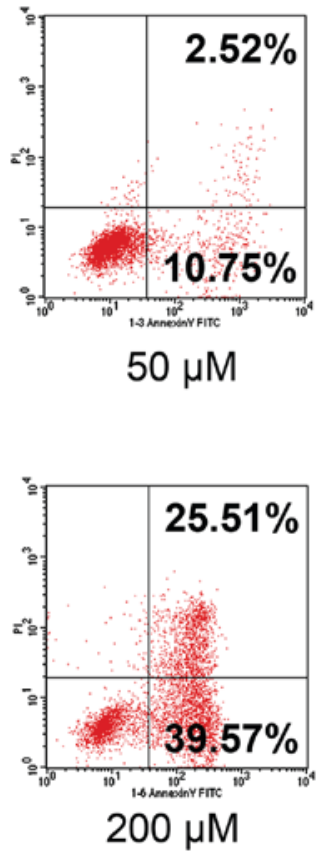

B

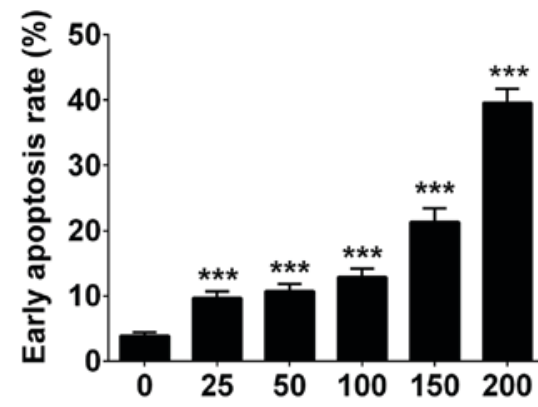

Drug concentration $(\mu \mathrm{M} / \mathrm{l})$

C
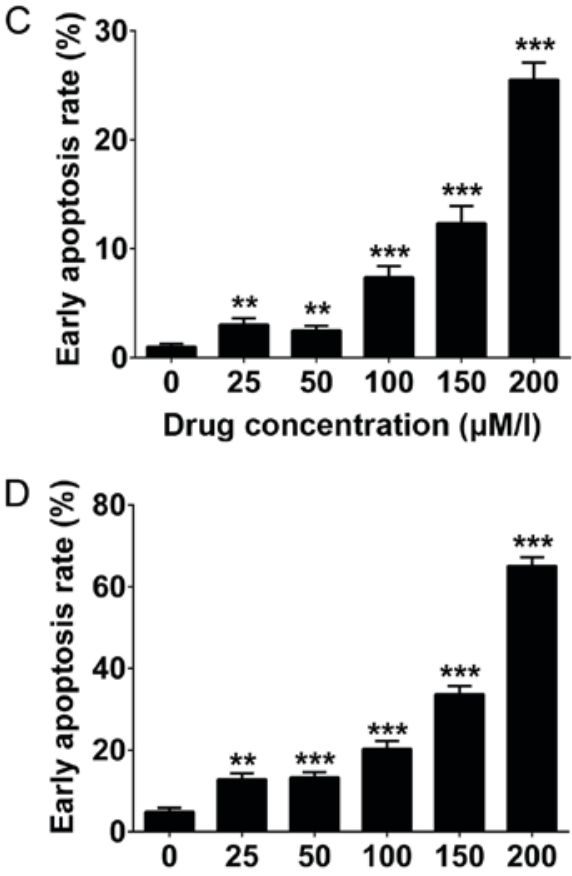

Drug concentration $(\mu \mathrm{M} / \mathrm{l})$

Figure 2. Apoptosis of THP-1 cells following DHA treatment. (A) Flow cytometry-based assessment of apoptosis in THP-1 cells with treated with various concentrations of DHA. (B) Early apoptosis rates of THP-1 cells. (C) Late apoptosis rates of THP-1 cells. (D) Total apoptosis rates of THP-1 cells. Data are presented as the mean \pm standard deviation. ${ }^{* * *} \mathrm{P}<0.001,{ }^{* * *} \mathrm{P}<0.01$. All data were obtained from at least three independent experiments. DHA, dihydroartemisinin. 
Table I. Sequences of primers used in reverse transcription-quantitative polymerase chain reaction.

\begin{tabular}{lll}
\hline Gene & Direction & \multicolumn{1}{c}{ Primer sequence (5'-3') } \\
\hline Akt1 & $\begin{array}{l}\text { Forward } \\
\text { Reverse }\end{array}$ & $\begin{array}{l}\text { ATGAGCGACGTGGCTATTGTGAAG } \\
\text { GAGCCGTCAGCCACAGTCTGGA } \\
\end{array}$ \\
& & TG \\
Akt2 & Forward & ATGAATGAGGTGTCTGTCATCAAA \\
& & GAAGGC \\
& Reverse & TGCTTGAGGCTGTTGGCGACC \\
Akt3 & Forward & CAGTCTGTCTGCTACAGCCTGGATA \\
& Reverse & ATGAGCGATGTACCATTGT \\
Bcl-2 & Forward & GAACTGGGGGAGGATTGTGG \\
& Reverse & CCGTACAGTTCCACAAAGGC \\
Bax & Forward & CCAGAGGCGGGGTTCAT \\
& Reverse & GGAAAAAGACCTCTCGGGGG \\
$\beta$-actin & Forward & CCAACCGCGAGAAGATGA \\
& Reverse & CCAGAGGCGTACAGGGATAG \\
\end{tabular}

ERK, extracellular-signal-regulated kinase; Akt, protein kinase B; Bcl-2, B-cell lymphoma 2; Bax, Bcl-2-associated X protein.

blocked by $5 \%$ skimmed milk suspended in Tris-buffered saline with Tween-20 for $1 \mathrm{~h}$ at room temperature. Each membrane was incubated with appropriate primary antibodies against p-Akt, t-Akt, p-ERK, t-ERK, cleaved caspase- 3 and $\beta$-actin, with a dilution ratio of $1: 1,000$ at $4^{\circ} \mathrm{C}$ overnight. Blots were subsequently incubated with a horseradish peroxidase-conjugated secondary antibody for $1 \mathrm{~h}$ at room temperature. Protein bands were visualized using $\mathrm{X}$-ray films and an enhanced chemiluminescence detection system (GE Healthcare Life Sciences, Little Chalfont, UK). Positive immunoreactive bands were densitometrically quantified and normalized to $\beta$-actin. Adobe Photoshop (Creative Suite 5; Adobe Systems, Inc., San Jose, CA, USA) was used for densitometry.

Statistical analysis. SPSS software (version 19.0; IBM Corp., Armonk, NY, USA) was used to analyze the data. The differences between the experimental groups and controls were assessed using the Student's t-test or one-way analysis of variance as appropriate. The data are expressed as the mean \pm standard deviation. All data were obtained from at least three independent experiments. $\mathrm{P}<0.05$ was considered to indicate a statistically significant difference.

\section{Results}

THP-1 cell viability decreases following DHA treatment. To investigate the effects of DHA, a cell viability assay using CCK-8 was performed on THP-1 cells. The viability of THP-1 cells was inhibited following treatment with DHA, accompanied by the appearance of morphological characteristics of apoptosis (Fig. 1B). The inhibitory effect of DHA on the viability of THP-1 cells was markedly increased at higher DHA concentrations following $96 \mathrm{~h}$ of treatment $(\mathrm{P}<0.05$;
Fig. 1C). DHA may inhibit the proliferation of THP-1 cells in a dose- and time-dependent manner.

DHA induces the apoptosis of THP-1 cells. As presented in Fig. 2, flow cytometry analysis was used to evaluate DHA-induced apoptosis. DHA significantly increased the total apoptosis rate (4.9 control vs. 12.77, 13.24, 20.28, 33.68 and $65.08 \%$ when treated with 25, 50, 100, 150 and $200 \mu \mathrm{M}$ $\mathrm{DHA}$, respectively; $\mathrm{P}<0.05$; Fig. $2 \mathrm{~A}$ and $\mathrm{D}$ ), and quantitative data regarding the early and late apoptosis rate were consistent with this (Fig. 2B and C). The results of the present study validated the inhibitory function of DHA functioned via the activation of the apoptosis pathway in THP-1 cells.

Downstream gene expression. To investigate the molecular mechanisms underlying the inhibitory effects of DHA in THP-1 human monocytic leukemia cells, RT-qPCR was used to examine variations in gene expression. It was demonstrated that the gene expression levels of Akt1, Akt2, Akt3 and B-cell lymphoma 2 (Bcl-2) were decreased in DHA-treated THP-1 cells in a dose-dependent manner, whereas the expression of Bcl-2-associated X protein (Bax) was upregulated (Fig. 3). The results indicated that these genes are potential downstream targets of DHA in monocytic leukemia treatment.

Akt, ERK and cleaved caspase-3 are potential downstream targets of DHA. Western blot analysis was used to determine the protein expression levels of phospho (p)-Akt/total-Akt, p-ERK/total-ERK and cleaved caspase-3 in THP-1 cells, following different concentrations of DHA treatment. The results revealed that the levels of p-Akt and p-ERK with increasing concentrations of DHA (Fig. 4A-C). However, DHA increased the levels of cleaved caspase-3 in a dose-dependent manner (Fig. 4A and D). Therefore, the results suggested that Akt, ERK and cleaved caspase-3 are potential downstream targets of DHA-induced apoptosis in THP-1 cells.

\section{Discussion}

Induction failure and a high incidence of relapse due to drug resistance are the principal problems surrounding AML treatment $(2,18)$. The use of novel drugs is one approach for treating patients who are resistant to standard therapies (19). Clinical evaluation of potentially effective drugs is essential to cancer chemotherapy, and may improve the prognosis of patients with refractory leukemia (20). AMoL is a rare but distinct disease, the increasing number of monocytes throughout its clinical course characterizes the disease, and cytogenetic characterization is required for diagnosis and prognosis stratification $(21,22)$. Thus, there is a requirement to identify less toxic and more efficacious treatment alternatives. As a result, increasing attention has been focused on the application of natural products in the treatment of AMoL (3).

DHA, one of the bioactive derivatives of artemisinin, has been investigated for the treatment of certain tumor types; Professor Tu YouYou, who discovered DHA, was awarded the Nobel Prize in $2015(23,24)$. A number of additional pharmacological effects of DHA have been identified, including 

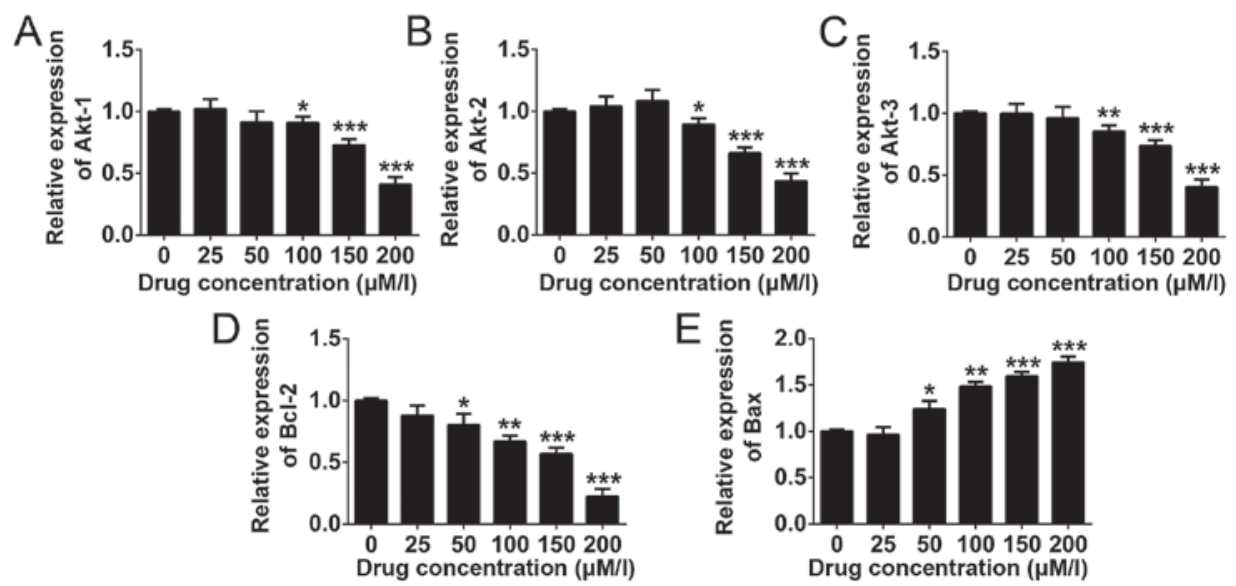

Figure 3. Apoptosis-associated gene expression in THP-1 cells following DHA treatment. RT-qPCR was employed to detect the expression of (A) Akt1, (B) Akt2, (C) Akt3, (D) Bcl-2 and (E) Bax in DHA-treated THP-1 cells. Data are presented as the mean \pm standard deviation. ${ }^{* * * *} \mathrm{P}<0.001,{ }^{* * *} \mathrm{P}<0.01,{ }^{*} \mathrm{P}<0.05$. All data were obtained from at least three independent experiments. RT-qPCR, reverse transcription quantitative polymerase chain reaction; Akt, protein kinase B; Bcl-2, B cell lymphoma 2; Bax, Bcl-2-associated X protein; DHA, dihydroartemisinin.
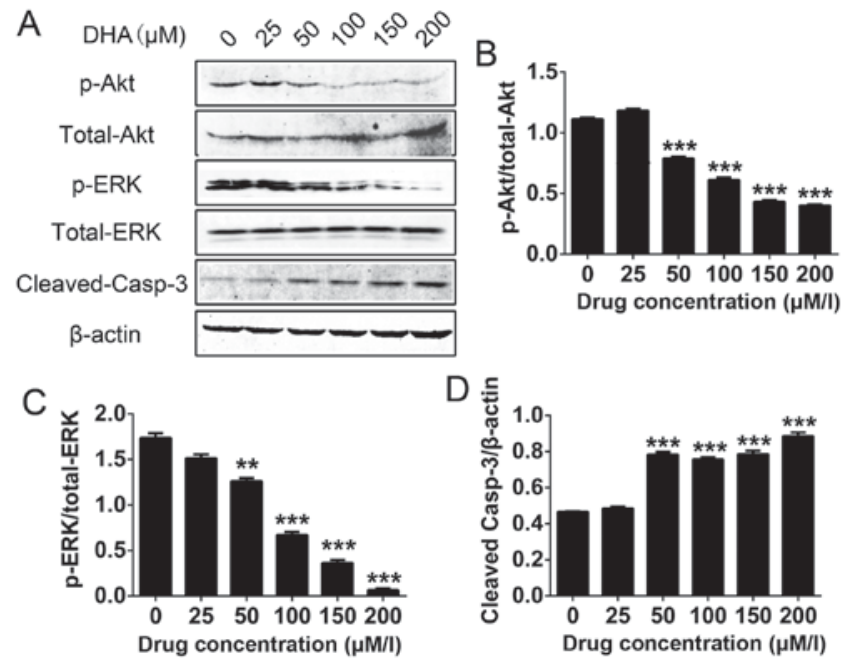

Figure 4. Western blots of p-t-Akt, p-ERK/t-ERK and cleaved caspase-3 levels following the DHA-induced apoptosis of THP-1 cells. (A) Expression of p/total-Akt, p-ERK/total-ERK and cleaved caspase-3 in THP-1 cells following DHA treatment. Levels of (B) p-t-Akt, (C) p-ERK/t-ERK and (D) cleaved caspase- 3 in THP-1 cells. Results are expressed as the ratio of cleaved caspase-3 to $\beta$-actin, p-Akt to t-Akt and p-ERK to t-ERK. Data are presented as the mean \pm standard deviation. ${ }^{* * * *} \mathrm{P}<0.001,{ }^{* * *} \mathrm{P}<0.01$. All data were obtained from at least three independent experiments. p-, phosphorylated; t-, total; DHA, dihydroartemisinin; Akt, protein kinase B; ERK, extracellular-signal-regulated kinase.

antitumor activity towards hepatocellular carcinoma in vitro and in vivo (25). Furthermore, DHA was identified to prevent breast cancer-induced osteolysis via inhibiting breast cancer cells and osteoclasts (26). For leukemia treatment, DHA can induce autophagy and inhibit the growth of iron-loaded human myeloid leukemia K562 cells via reactive oxygen species toxicity (27); DHA and its derivative induce apoptosis in acute myeloid leukemia cells through the Noxa-mediated pathway, requiring iron and an endoperoxide moiety (28). However, the effect of DHA on AMoL has yet to be fully elucidated.

The aim of the present study was to investigate whether DHA had antitumor activity against human AMoL. It was identified that DHA has a strong anti-leukemia effect in the THP-1 AMoL cell line in vitro. The results indicated that DHA inhibited the spontaneous growth of THP-1 cells in a time- and dose-dependent manner. To further investigate the mechanisms of DHA anti-leukemia activity, the effect of DHA was analyzed with regards to apoptosis and the activation of Akt/ERK survival signaling and Bcl-2/Bax/caspase-3 apoptosis pathways that are constitutively expressed in THP-1 cells. Results of the present study indicated that DHA-induced apoptosis was caused by downregulating Akt/ERK signaling and activating the caspase-3 pathway, following the balance of the Bcl-2/Bax axis.

Distinct from normal cells, leukemia cells often express constitutively active survival-signaling pathways, such as Akt and ERK among others, due to gene mutations, rearrangements and chromosomal translocations; these survival signaling pathways serve vital roles in tumorigenesis, proliferation, anti-apoptosis and drug resistance (29-31). The higher the number of constitutively active growth signaling pathways in acute myelogenous leukemia, the poorer the prognosis. Results indicated that, in the acute monoblastic leukemia cell line THP-1, the Akt and ERK signal transduction pathways were suppressed simultaneously, and that the caspase-3 apoptosis signaling pathway was activated by the downregulation of the Bcl-2/Bax ratio following DHA treatment. It was speculated that DHA-mediated inhibition of Akt/ERK pathway activation and the promotion of caspase-3 activation would result in a more effective response to anti-leukemia therapy, as the inhibitors would simultaneously target three pathways in THP-1 cells.

A number of specific inhibitors target a single signaling molecule in the treatment of leukemia; however, DHA may be more effective compared with common inhibitors as drug resistance frequently emerges following the hyperactivation of alternative signaling pathways under treatment of a single target. It is predicted that DHA-resistance in AMoL may rarely occur, the response to DHA may be increased and response duration may be longer as DHA exhibited multi-targeting characteristics. Inhibition of multiple signaling pathways increases the therapeutic ability of DHA. 
In the present study, the role of DHA in restricting the proliferation and inducing apoptosis in $\mathrm{AMoL}$ cells was investigated. DHA may directly or indirectly affect the activation of Akt/ERK survival signaling and caspase-3 apoptosis signaling pathways, all of which are key regulators of cell survival and apoptosis, particularly during AMoL treatment $(32,33)$. However, additional in-depth investigations must follow this preliminary study. First, the intermediate molecular mechanisms underlying DHA-mediated changes to cell survival and apoptosis signaling pathways must be clarified. Secondly, in vivo experiments must be carried out to verify the treatment effects of DHA. Finally, the results must be validated through clinical trials and applications.

In conclusion, the present study indicated that DHA exerted effective antitumor activities against $\mathrm{AMoL}$ in vitro. Furthermore, DHA downregulated the expression of p-Akt and p-ERK, whilst also upregulating the protein expression of cleaved caspase-3 through increasing the Bcl-2/Bax ratio. This may be one mechanism by which DHA exerts its effects in AMoL. However, further and more comprehensive studies are required to confirm this.

\section{Acknowledgements}

The present study was supported by grants from the Shanghai Health Development Planning Commission (grant no. ZY3-CCCX-3-3006), the National Natural Science Foundation of China (grant nos. 81500392 and 31201010), the Shanghai Committee of Science and Technology of China (grant nos. 12ZR1419500 and 114119a8700) and the Shanghai Health Bureau (grant no. ZYSNXD-CCZDYJ029).

\section{References}

1. Ommen HB: Monitoring minimal residual disease in acute myeloid leukaemia: A review of the current evolving strategies. Ther Adv Hematol 7: 3-16, 2016.

2. Peng H, Wang H, Xue P, Hou Y, Dong J, Zhou T, Qu W, Peng S, Li J, Carmichael PL, et al: Suppression of NRF2-ARE activity sensitizes chemotherapeutic agent-induced cytotoxicity in human acute monocytic leukemia cells. Toxicol Appl Pharmacol 292: $1-7,2016$

3. Guo Y, Shan Q, Gong Y, Lin J, Shi F, Shi R and Yang X: Curcumin induces apoptosis via simultaneously targeting AKT/mTOR and $\mathrm{RAF} / \mathrm{MEK} / \mathrm{ERK}$ survival signaling pathways in human leukemia THP-1 cells. Pharmazie 69: 229-233, 2014.

4. Ozpolat B, Akar U, Steiner M, Zorrilla-Calancha I, Tirado-Gomez M, Colburn N, Danilenko M, Kornblau S and Berestein GL: Programmed cell death-4 tumor suppressor protein contributes to retinoic acid-induced terminal granulocytic differentiation of human myeloid leukemia cells. Mol Cancer Res 5: 95-108, 2007.

5. Murashige N, Tabanda R and Zalusky R: Occurrence of acute monocytic leukemia in a case of untreated Waldenstrom's macroglobulinemia. Am J Hematol 71: 94-97, 2002.

6. Cline MJ: Histiocytes and histiocytosis. Blood 84: 2840-2853, 1994.

7. Kumar RK, Basu S, Lemke HD, Jankowski J, Kratz K, Lendlein A and Tetali SD: Effect of extracts of poly(ether imide) microparticles on cytotoxicity, ROS generation and proinflammatory effects on human monocytic (THP-1) cells. Clin Hemorheol Microcirc 61: 667-680, 2016.

8. Li Y: Qinghaosu (artemisinin): Chemistry and pharmacology. Acta Pharmacol Sin 33: 1141-1146, 2012.

9. Lin AJ, Klayman DL and Milhous WK: Antimalarial activity of new water-soluble dihydroartemisinin derivatives. J Med Chem 30: 2147-2150, 1987.
10. Ashley EA, McGready R, Hutagalung R, Phaiphun L, Slight T, Proux S, Thwai KL, Barends M, Looareesuwan S, White NJ and Nosten F: A randomized, controlled study of a simple, once-daily regimen of dihydroartemisinin-piperaquine for the treatment of uncomplicated, multidrug-resistant falciparum malaria. Clin Infect Dis 41: 425-432, 2005.

11. Gordi T and Lepist EI: Artemisinin derivatives: Toxic for laboratory animals, safe for humans? Toxicol Lett 147: 99-107, 2004.

12. Mi YJ, Geng GJ, Zou ZZ, Gao J, Luo XY, Liu Y, Li N, Li CL, Chen YQ, Yu XY and Jiang J: Dihydroartemisinin inhibits glucose uptake and cooperates with glycolysis inhibitor to induce apoptosis in non-small cell lung carcinoma cells. PLoS One 10: e0120426, 2015.

13. Liu W, Wang DW, Yu SY, Cao Y, Yang L, E XQ, Yao GJ and Bi ZG: The effect of dihydroartemisinin on the proliferation, metastasis and apoptosis of human osteosarcoma cells and its mechanism. J Biol Regul Homeost Agents 29: 335-342, 2015.

14. Feng $\mathrm{X}$, Li L, Jiang $\mathrm{H}$, Jiang $\mathrm{K}$, Jin $\mathrm{Y}$ and Zheng J: Dihydroartemisinin potentiates the anticancer effect of cisplatin via mTOR inhibition in cisplatin-resistant ovarian cancer cells: Involvement of apoptosis and autophagy. Biochem Biophys Res Commun 444: 376-381, 2014

15. Park J, Lai HC, Singh M, Sasaki T and Singh NP: Development of a dihydroartemisinin-resistant Molt-4 leukemia cell line. Anticancer Res 34: 2807-2810, 2014.

16. Chan HW, Singh NP and Lai HC: Cytotoxicity of dihydroartemisinin toward Molt-4 cells attenuated by N-tert-butyl-alpha-phenylnitrone and deferoxamine. Anticancer Res 33: 4389-4393, 2013.

17. Schmittgen TD and Livak KJ: Analyzing real-time PCR data by the comparative C(T) method. Nat Protoc 3: 1101-1108, 2008.

18. Zhang D, Wang Q, Zhu T, Cao J, Zhang X, Wang J, Wang X, Li Y, Shen B and Zhang J: RACK1 promotes the proliferation of THP1 acute myeloid leukemia cells. Mol Cell Biochem 384: 197-202, 2013

19. Zeng C, Wang W, Yu X, Yang L, Chen S and Li Y: Pathways related to PMA-differentiated THP1 human monocytic leukemia cells revealed by RNA-Seq. Sci China Life Sci 58: 1282-1287, 2015.

20. Evans FJ and Hilton JH: Polymyositis associated with acute Nonocytic leukemia: Case report and review of the literature. Can Med Assoc J 91: 1272-1275, 1964.

21. Wang Y, Ma L, Wang C, Sheng G, Feng L and Yin C: Autocrine motility factor receptor promotes the proliferation of human acute monocytic leukemia THP-1 cells. Int J Mol Med 36: 627-632, 2015 .

22. Jain SK, Sahu R, Walker LA and Tekwani BL: A parasite rescue and transformation assay for antileishmanial screening against intracellular Leishmania donovani amastigotes in THP1 human acute monocytic leukemia cell line. Journal of visualized experiments 70: e4054, 2012.

23. Tu YY: TU You-you won Lasker Debakey clinical medical research award-for her outstanding achievements in studies on artemisinin. Zhongguo Zhong Xi Yi Jie He Za Zhi 31: 1301, 2011 (In Chinese)

24. Dong YJ, Li WD and Tu YY: Effect of dihydro-qinghaosu on auto-antibody production, TNF alpha secretion and pathologic change of lupus nephritis in BXSB mice. Zhongguo Zhong Xi Yi Jie He Za Zhi 23: 676-679, 2003 (In Chinese).

25. Zhang CZ, Zhang H, Yun J, Chen GG and Lai PB: Dihydroartemisinin exhibits antitumor activity toward hepatocellular carcinoma in vitro and in vivo. Biochem Pharmacol 83: 1278-1289, 2012

26. Feng MX, Hong JX, Wang Q, Fan YY, Yuan CT, Lei XH, Zhu M, Qin A, Chen HX and Hong D: Dihydroartemisinin prevents breast cancer-induced osteolysis via inhibiting both breast cancer cells and osteoclasts. Sci Rep 6: 19074, 2016.

27. Wang $\mathrm{Z}, \mathrm{Hu} \mathrm{W}$, Zhang JL, Wu XH and Zhou $\mathrm{HJ}$ : Dihydroartemisinin induces autophagy and inhibits the growth of iron-loaded human myeloid leukemia K562 cells via ROS toxicity. FEBS Open Bio 2: 103-112, 2012.

28. Zhao X, Zhong H, Wang R, Liu D, Waxman S, Zhao L and Jing Y: Dihydroartemisinin and its derivative induce apoptosis in acute myeloid leukemia through Noxa-mediated pathway requiring iron and endoperoxide moiety. Oncotarget 6: $5582-5596,2015$.

29. Lim SG, Suk K and Lee WH: Reverse signaling from LIGHT promotes pro-inflammatory responses in the human monocytic leukemia cell line, THP-1. Cell Immunol 285: 10-17, 2013. 
30. Teng CL, Han SM, Wu WC, Hsueh CM, Tsai JR, Hwang WL and Hsu SL: Mechanistic aspects of lauryl gallate-induced differentiation and apoptosis in human acute myeloid leukemia cells. Food Chem Toxicol 71: 197-206, 2014.

31. Shi D, Xu Y, Du X, Chen X, Zhang X, Lou J, Li M and Zhuo J: Co-treatment of THP-1 cells with naringenin and curcumin induces cell cycle arrest and apoptosis via numerous pathways. Mol Med Rep 12: 8223-8228, 2015.

32. Olesen LH, Aggerholm A, Andersen BL, Nyvold CG, Guldberg P, Nørgaard JM and Hokland P: Molecular typing of adult acute myeloid leukaemia: Significance of translocations, tandem duplications, methylation, and selective gene expression profiling. Br J Haematol 131: 457-467, 2005.
33. Ruvolo PP, Qiu Y, Coombes KR, Zhang N, Neeley ES, Ruvolo VR, Hail N Jr, Borthakur G, Konopleva M, Andreeff M and Kornblau SM: Phosphorylation of GSK $3 \alpha / \beta$ correlates with activation of AKT and is prognostic for poor overall survival in acute myeloid leukemia patients. BBA Clin 4: 59-68, 2015. 